\title{
47 Tucanae: the Spectroscopic versus CMD Age Discrepancy
}

\author{
Alexandre Vazdekis \\ Instituto de Astrofísica de Canarias, La Laguna 38200, Tenerife, Spain. \\ E-mail:vazdekis@ll.iac.es \\ Maurizio Salaris \\ Liverpool John Moores University, Twelve Quays House, Egerton \\ Wharf, Birkenhead CH41 1LD, U.K. \\ Nobuo Arimoto \\ Institute of Astronomy, University of Tokyo, Osawa 2-21-1, Mitaka, \\ Tokyo 181-0015, Japan \\ James A. Rose \\ Department of Physics and Astronomy, University of North Carolina, \\ Chapel Hill, NC 27599, U.S.A.
}

\begin{abstract}
.
We investigate the origin of the discrepancy between the age estimates based on the Color-Magnitude-Diagram and the integrated spectra of 47 Tuc. Using a newly defined $\mathrm{H} \gamma$ age indicator, with unprecedented power to disentangle the degeneracy between age and metallicity, we confirm the very large spectroscopic age derived for this cluster. We explore a number of key parameters with potential to decrease the Balmer lines and show the necessity of turnoff stars with lower temperatures. We find that alpha-enhanced isochrones with atomic diffusion leads to a younger spectroscopic age in better agreement with the CMD age.
\end{abstract}

\section{Introduction}

Globular cluster age determination represents one of the key topics of this meeting. Two approaches are followed to achieve this aim: if the stellar populations are resolved into individual stars and Color-Magnitude Diagrams (CMD) are plotted it is possible to derive the age from the TO luminosity once the distance is fixed. This approach can be applied to many clusters of the Galaxy. The extragalactic clusters, whose stellar populations cannot be resolved, should be studied through their integrated light with the help of stellar population synthesis models. These models make use of theoretical isochrones, which are converted to the observational plane (i.e., fluxes, colours, spectra) on the basis of empirical or theoretical stellar libraries, and integrated along the isochrone. Whereas the CMD-derived ages may be sensitive to the dating method employed (e.g., 
Alonso et al. 1997) and to the input physics of the theoretical isochrones used as a reference (e.g., Salaris \& Weiss 1998), the second approach must deal with the fundamental age-metallicity degeneracy, which affects not only integrated colors but also absorption-features including the Balmer lines (Worthey 1994).

Recently, new age-dating techniques based on Balmer lines at high resolution (Jones \& Worthey 1995; Vazdekis \& Arimoto 1999; Vazdekis et al. 2001) have shown great promise in untangling the age-metallicity degeneracy. These techniques should be tested and calibrated on metal-rich Galactic globular clusters for which CMD-derived ages are feasible. These tests have revealed two major concerns: i) the obtained ages are unreasonably large (>20 Gyr) (Jones 1999; Gibson et al. 1999; Vazdekis \& Arimoto 1999) and ii) a severe disagreement is found between the spectroscopic and CMD ages of 47 Tuc (Gibson et al. 1999), the latter in the range 9-13 Gyr (Salaris \& Weiss 1998; Liu \& Chaboyer 2000). This discrepancy shows that current stellar population synthesis models used for interpreting the integrated light of stellar systems may have severe zero point problems and that only relative ages are meaningful.

5 the spectroscopic age of 47 Tuc. In $\S 4$ we discuss possible causes for this 5 discrepancy and in $\S 5$ we summarize our conclusions.

\section{New tools for the analysis of the mean luminosity-weighted ages}

We make use of the evolutionary stellar population synthesis model of Vazdekis (1999), which predicts spectral energy distributions (SEDs) in the optical wavelength range for single burst old-aged stellar populations of different metallicities at resolution $1.8 \AA$ (FWHM). The standard isochrones used by this code are those of Girardi et al. (2000), which assume solar ratio abundance trends. These isochrones are transformed to the observational plane on the basis of extensive empirical (rather than theoretical) photometric (e.g., Alonso et al. 1996 and 1999) and spectral (Jones 1999) stellar libraries. These model SEDs represent an important improvement over previous models (e.g., Worthey 1994; Vazdekis et al. 1996), which only predicted the strengths of a number of strong features using fitting functions which relate the feature strengths to stellar atmospheric parameters, under a particular resolution and instrumental configuration (Worthey et al. 1994; Worthey \& Ottaviani 1997).

On the basis of these models Vazdekis et al. (2001) defined a new index $\left(\mathrm{H} \gamma_{\sigma<130}\right)$, centered on $\mathrm{H} \gamma$, which provides unprecedented power at breaking the age-metallicity degeneracy. Unlike $\mathrm{H} \gamma$ indices of Rose (1994) and Jones \& Worthey (1995), $\mathrm{H} \gamma_{\sigma<130}$ is insensitive to metallicity (for $-0.7 \leq \log \left(Z / Z_{\odot}\right) \leq$ +0.2 ) (see left plot of Fig. 1) and spectral resolution (for $\sigma<130 \mathrm{kms}^{-1}$ ).

\section{Spectroscopic age estimate of 47 Tucanae}

Fig. 1 shows the 47 Tuc value using a very high S/N spectrum of Rose (1994). The error bar size along the age axis represents the maximum range for the most recent CMD age estimates $(9 \pm 1$ Gyr in Salaris \& Weiss 1998 and $12.5 \pm$ 1.5 Gyr in Liu \& Chaboyer 2000). The right panels show the unprecedented agemetallicity disentangling power achieved when plotting this index versus various metallicity indicators. All the model indices were measured directly on Vazdekis 

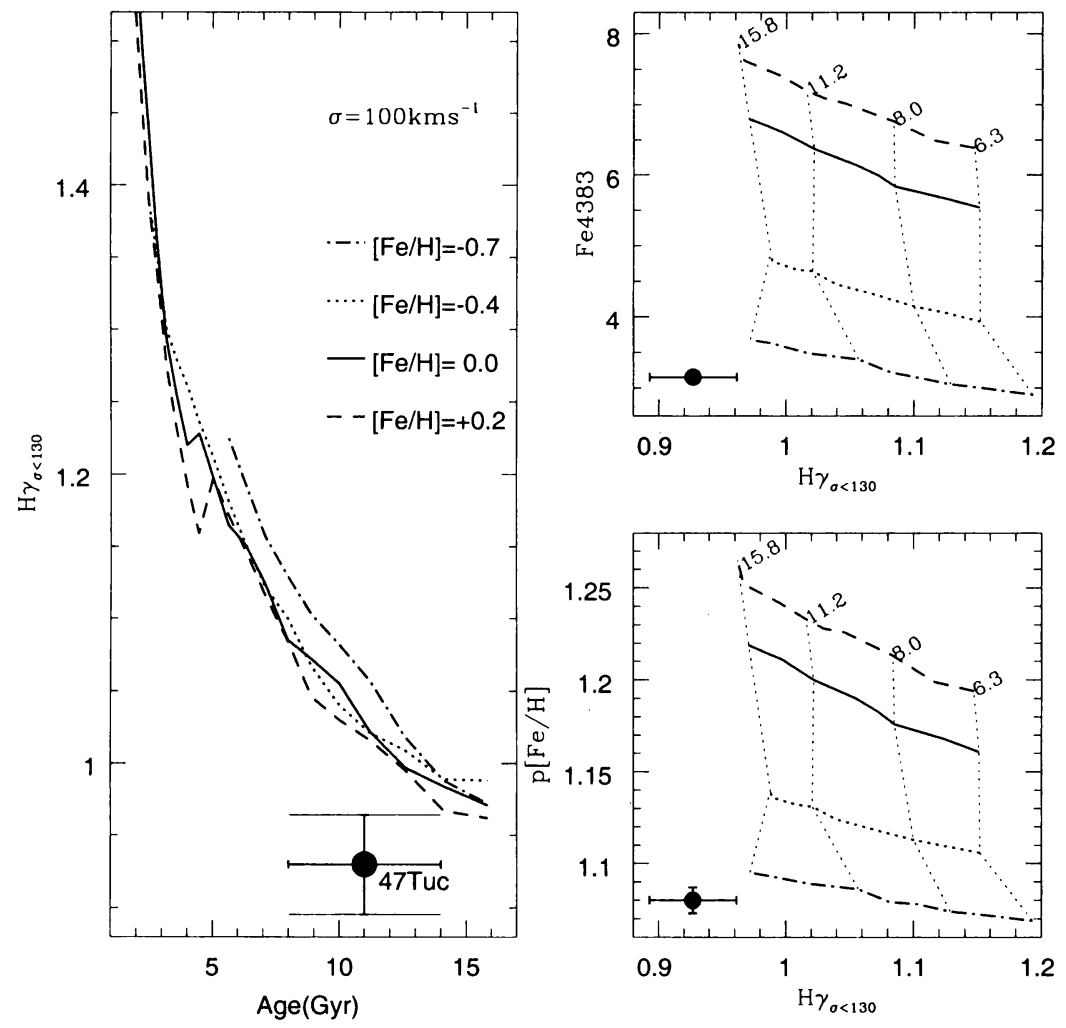

Figure 1. Left: $\mathrm{H} \gamma_{\sigma<130}$ age indicator measured on Vazdekis (1999) SEDs smoothed to $\sigma \sim 100 \mathrm{kms}^{-1}$ to match the resolution of the spectrum of 47 Tuc (Rose 1994). The error bar size along the age axis represents the maximum range for the most recent CMD ages $(9 \pm 1 \mathrm{Gyr}$ in Salaris \& Weiss 1998 and $12.5 \pm 1.5$ Gyr in Liu \& Chaboyer 2000), and therefore we plot 47 Tuc at 11 Gyr. Right: various metallicity indicators, Fe4383 and $\mathrm{p}[\mathrm{Fe} / \mathrm{H}]$ (Worthey et al. 1994 and Rose 1994, respectively), plotted vs. $\mathrm{H} \gamma_{\sigma<130}$. Line types as in the left plot, while thin dotted lines mean models of equal ages (indicated in Gyr). 


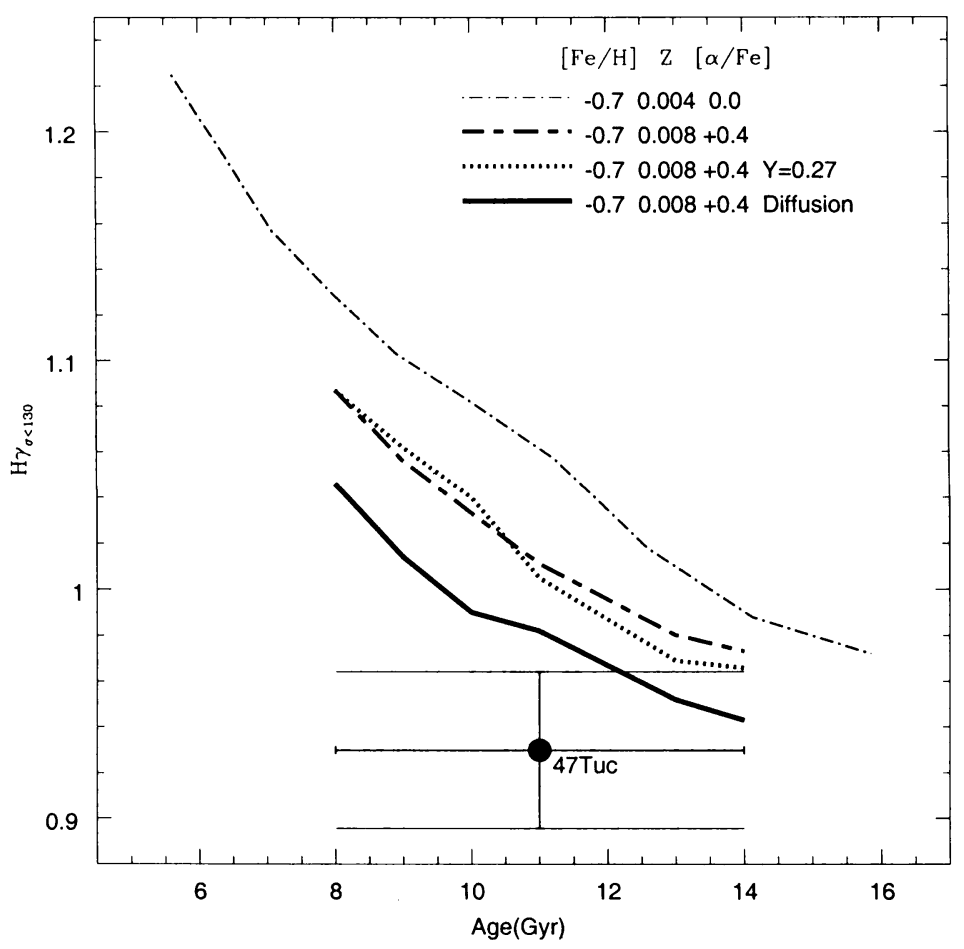

Figure 2. $\mathrm{H} \gamma_{\sigma<130}$ measured on the integrated spectra synthesized on the basis of different isochrones.

(1999) SEDs smoothed to match the resolution of the spectrum of 47 Tuc. All these plots suggest ages well in excess of $15 \mathrm{Gyr}$, confirming the Gibson et al. (1999) result. These authors used different spectra of 47 Tuc as well as a different population synthesis code (Worthey 1994) and $\mathrm{H} \gamma$ age indicator (Jones \& Worthey 1995).

The possibility that nebular emission could be partially filling in $\mathrm{H} \gamma$ feature, which translates to lower $\mathrm{H} \gamma_{\sigma<130}$ index values and therefore larger ages, is discarded by Vazdekis et al. (2001) since the derived age for 47 Tuc is essentially independent of the Balmer line used (emission would affect them by differing amounts), and since other metal-rich Galactic globular clusters show the same "anomalous" behavior in age as 47 Tuc.

\section{Looking for the origin of the age discrepancy}

The Balmer indices are dominated by the hottest stars along the isochrone (e.g., Buzzoni, Mantegazza \& Gariboldi 1994), i.e., by turnoff (TO) and horizontalbranch (HB) stars. For a GC with a red clump such as 47 Tuc, we have verified that decreasing the $\mathrm{HB}$ star's temperature by $150 \mathrm{~K}$ yields $\mathrm{H} \gamma_{\sigma<130}$ val- 
ues smaller by less than $0.01 \AA$ (for large ages). Therefore we have to look for those parameters that would decrease the TO temperature, in order to decrease $\mathrm{H} \gamma_{\sigma<130}$ model predictions.

Spectroscopic determinations in individual stars of this cluster have confirmed the enhancement of $\alpha$-elements (e.g., Carney 1996). Salaris \& Weiss (1998) have shown that when comparing $\alpha$-enhanced with scaled-solar isochrones the former have a hotter TO if the comparison is made at the same global metallicity $(\mathrm{Z}=0.008)$, while if the comparison is made at the same $[\mathrm{Fe} / \mathrm{H}]=-0.7$, they have a cooler TO. This result is confirmed by VandenBerg, et al. (2000). Fig. 2 shows that the implementation in our population synthesis code of these $\alpha$ enhanced isochrones $(<[\alpha / \mathrm{Fe}]>=0.4)$, transformed to the observational plane following our empirical prescriptions (the same ones that we applied to the Girardi et al. 2000 isochrones), provides lower $\mathrm{H} \gamma_{\sigma<130}$ values, and yields considerably younger age estimates (although not as young as the CMD-ages).

Fig. 2 shows that the spectroscopic age is not affected significantly by increasing the initial He content (from $\mathrm{Y}=0.254$ to 0.273 ) of our $\alpha$-enhanced isochrones.

Atomic diffusion is capable of changing the TO temperature of low mass stars at a given age (e.g., Salaris, Groenewegen \& Weiss 2000). For a given initial chemical composition and age, TO temperatures are significantly cooler than in isochrones without diffusion. Fig. 2 shows that the $\mathrm{H} \gamma_{\sigma<130}$ measurement for the model spectra computed on the basis of isochrones calculated following Salaris et al.(2000) and the same input physics and $\alpha$-enhancement as in Salaris \& Weiss (1998), yield ages in much better agreement with the CMD (see Vazdekis et al. 2001 for an extended discussion).

\section{General Conclusions}

Current age estimates based on the integrated spectra of old stellar populations do suffer from a zero point problem, which is seen when compared to the CMD ages, making only relative ages secure. The input physics of the evolutionary models should be improved, including the well known main sequence. We find that $\alpha$-enhanced isochrones with atomic diffusion seem to work in the right direction for 47 Tuc. Finally, the CMD versus spectroscopic age tests should be extended to metal-poor clusters.

\section{References}

Alonso, A., Arribas, S. \& Martínez-Roger, C. 1999, A\&AS, 140, 261

Alonso, A., Salaris, M., Martínez-Roger, C., Straniero, O. \& Arribas, S. 1997, A\&A, 323, 374

Alonso, A., Arribas, S. \& Martínez-Roger, C. 1996, A\&A, 313, 873

Buzzoni, A., Mantegazza, L. \& Gariboldi, G. 1994, AJ, 107, 513

Carney, B.W. 1996, PASP, 108, 900

Gibson, B., Madgwick, D., Jones, L.A., Da Costa, G., Norris, J. 1999, AJ, 118, 1268 
Girardi, L., Bressan, A., Bertelli, G. \& Chiosi, C. 2000, A\&AS, 141, 371

Jones, L.A. 1999, Ph.D. Thesis, Univ. North Carolina, Chapel Hill

Jones, L.A. \& Worthey, G. 1995, ApJ, 446, L31

Liu, W. M. \& Chaboyer, B. 2000, ApJ, 544, 818

Rose, J.A. 1994, AJ, 107, 206

Salaris, M., Groenewegen, M. \& Weiss, A. 2000, A\&A, 355, 299

Salaris, M. \& Weiss, A. 1998, A\&A, 335, 943

VandenBerg, D., Swenson, F. \& Alexander, D. 2000, ApJ, 532, 430

Vazdekis, A. 1999, ApJ, 513, 224

Vazdekis, A., Salaris, M., Arimoto, N. \& Rose, J. 2001, ApJ, 549, 274

Vazdekis, A. \& Arimoto, N. 1999, ApJ, 525, 144

Vazdekis, A., Casuso, E., Peletier, R. F. \& Beckman, J. E. 1996, ApJS, 106, 307

Worthey, G. 1994, ApJS, 95, 107

Worthey, G. \& Ottaviani, D.L. 1997, ApJS, 111, 377

Worthey, G., Faber, S., González, J. \& Burstein, D. 1994, ApJS, 94, 687

\section{Discussion}

J. Hesser: Could you explain how the 47 Tuc spectrum was obtained?, How do you know that your spectrum is representative of the stellar population of the cluster and is not being affected by the contribution of a few peculiar stars?

A. Vazdekis: This spectrum was obtained by Jim Rose and you can find all the technical details in Rose (1994). Basically, the telescope scans back and forth in different regions of the cluster. We obtain similar results with another spectrum of this cluster obtained with a different telescope and instrumental configuration and different scanned regions. The results that I have shown here are in full agreement with Gibson et al. (1999), who used different spectra.

G. Bruzual: Did you apply this age analysis to another cluster?

A. Vazdekis: We have not applied such a detailed analysis to other clusters. However, we tested various metal-rich clusters with well known CMD ages, and for which either $\mathrm{H} \gamma_{\sigma<130}$ or different Balmer lines can be measured. We obtain very similar results and 47 Tuc does not seem to be a peculiar cluster in this respect. We are planning to extend this analysis to globulars of lower metallicity.

D. Alloin: The spectroscopic age determination depends on the completeness of the stellar libraries used (at each metallicity). How does this completeness change across the metallicity range?

A. Vazdekis: The library we are using for this purpose has a good stellar parameter coverage in the range $-0.8 \leq[\mathrm{Fe} / \mathrm{H}] \leq+0.2$ (see Jones 1999, Vazdekis 1999). 